Keywords: Immunotherapy; cytotoxic T-lymphocyte antigen 4 (CTLA-4); programmed death-1 (PD-1); melanoma; checkpoint blockade; toxicity

\title{
Exploiting CTLA-4, PD-1 and PD-L1 to reactivate the host immune response against cancer
}

\author{
S A Quezada ${ }^{1}$ and K S Peggs ${ }^{*, 1}$ \\ ${ }^{1}$ Cancer Immunology Unit, Department of Haematology, UCL Cancer Institute, Paul O'Gorman Building, \\ 72 Huntley Street, London WC1E 6BT, UK
}

\begin{abstract}
The past few years have witnessed something of a renaissance in the field of cancer immunotherapy, relating largely to the clinical advances that have been associated with the development of monoclonal antibodies targeting the immune inhibitory coreceptors CTLA-4 and PD-1 and to the pursuit of genetically modified antigen-redirected adoptive T-cell therapies. These advances are based on a more substantial understanding of the factors restricting effective immune therapies that has been derived from the study of pre-clinical models of tumour growth in immune competent mice. Just as the recognition of the importance of positive co-stimulatory signaling has been instrumental to recent advances in the development of genetically modified antigen-specific adoptive cellular therapies, an increasing awareness of the ability of tumours to subvert multiple immune inhibitory pathways, effectively blunting the development or expansion of any anti-tumour immunity, is fostering the development of novel therapies that appear active as monotherapies but may achieve their greatest impact in combinatorial regimens. This mini-review will focus on attempts to target co-inhibitory members of the immunoglobulin superfamily.
\end{abstract}

It is generally held that key advances in the development of effective immunotherapies will derive both from a better biological understanding of the immunological responses occurring during tumour induction and growth and how the critical components of such responses fit together, and a deeper understanding of the processes underlying malignant transformation and cancer progression. Although these two fields of cancer immunology and basic cancer biology have often developed along divergent pathways, the overlap is now extending. Data supporting the validity and aiding refinement of such key concepts as immune surveillance and immune editing have furthered our understanding of the dynamic nature of the relationship between host and tumour. The basic idea that the immune system is capable of recognising and responding to cancer has been expounded for over 150 years, with early speculation that the incidence of cancer would be much greater were it not for the ability of the immune system to identify and eliminate nascent tumour cells. This concept later become known as the immune surveillance hypothesis (Burnet, 1967). Direct supportive evidence, however, remained elusive for all but virus-associated malignancies, and the idea was controversial until 2001, when a landmark paper by Robert Schreiber in collaboration with Lloyd Old demonstrated that lymphocytes and the immune stimulator interferon- $\gamma$ cooperate to inhibit the development of spontaneous and carcinogen-induced tumours in mice genetically engineered to lack a functional immune system (RAG-2 $2^{-1-}$; Shankaran et al, 2001). They also recognised that some tumour cells escaped detection and eventually caused cancer and proposed that the cellular composition of such tumours, driven by the selective pressure exerted by immune system, becomes serially less immunogenic (immunoediting), perhaps as a consequence of downregulation of the molecules that are required for immune recognition. Experimental support for this idea was provided by data demonstrating that tumour cells from immunodeficient mice were more immunogenic than those from immunocompetent mice. The central tenets of immunoediting have evolved slightly since inception but may be considered to consist of three processes occurring either independently or sequentially. Firstly, 'elimination' in which immunity functions as an extrinsic tumour suppressor (equivalent to the original concept of immunosurveillance); secondly, 'equilibrium' in which

*Correspondence: Dr KS Peggs; E-mail: k.peggs@cancer.ucl.ac.uk 
cancerous cells survive but are held in check by the immune system (Koebel et al, 2007); and thirdly 'escape' in which tumour cell variants with either reduced immunogenicity or the capacity to attenuate or subvert immune responses grow into clinically apparent cancers. These data raise a potentially formidable obstacle to the delivery of clinically useful immunotherapies, suggesting that by the time a cancer becomes detectable it is already beyond the capabilities of the host immune system to eradicate it. Critically from the therapeutic standpoint, however, it has been demonstrated that it is possible to make these 'edited' cells visible to the immune system by increasing their antigen expression or by manipulation of intrinsic or extrinsic regulators of immunity. A greater focus on the tumour microenvironment, and the immune mediators therein, has been coupled to advances in our understanding of the regulation of immune responses in general, leading to the development of novel therapeutic strategies. Although many components of the microenvironment merit consideration, including inhibitory macrophage/monocyte populations, optimisation of antigen presentation by dendritic cell populations, vascular activation and chemokine signatures controlling T-cell trafficking, and signaling via tumour necrosis factor receptor family members, we will focus on the role of members of the co-stimulatory immunoglobulin superfamily in shaping the anti-tumour immune response and how these might be manipulated for therapeutic gain.

In order to mitigate uncontrolled propagation of harmful immune responses, the immune system contains a multitude of inhibitory feedback loops. The most critical of these have been termed immunological checkpoints and they act to regulate the amplitude of immune responses, to provide a molecular shield protecting against collateral tissue damage that might occur during immune responses to infections and to maintain peripheral selftolerance. A number of these key checkpoints belong to the extended CD28 family of receptors and their ligands. This family contributes both co-stimulatory pathways (notably CD28 and ICOS), which are central to the development of productive immune responses, and co-inhibitory pathways (exemplified by CTLA-4 and PD-1, Figure 1). CTLA-4 is expressed exclusively on $\mathrm{T}$ cells and its ligands CD80 (B7.1) and CD86 (B7.2) restricted largely to professional antigen-presenting cells or other immune mediators, for example, $\mathrm{T}$ cells themselves. This strict compartmentalisation may help to regulate the powerful inductive impact co-stimulatory signaling has on immune responses and reflects the importance of CD28/CTLA-4 and their shared ligands in the early activation of productive immunity. Genetic ablation of CTLA-4 results in profound lymphoproliferation, particularly of the CD4 ${ }^{+}$ T-cell compartment, culminating in widespread tissue infiltration and death at approximately 3-4 weeks of age in CTLA-4 ${ }^{-1}$ mice (Chambers et al, 1997). PD-1 has a slightly broader expression profile, being present also on B cells and NK cells, though much of its physiological inhibitory function has been established in the context of T-cell responses. By contrast, its ligands (more notably PD-L1) have a much broader tissue distribution, being expressed on both haematopoietic cells (B, T, myeloid and dendritic cells) as well as non-haematopoietic cells, including microvascular endothelial cells and in non-lymphoid organs, including heart, lung, pancreas, muscle and placenta. This distribution suggests that interaction of receptors and ligands may be more important in regulating effector T-cell responses in peripheral tissues. Indeed, the upregulation of PD-L1 ligand in response to interferon- $\gamma$ provides one possible mechanism by which peripheral tissues might be protected from the collateral damage that could ensue from T-cell activation in the context of infection, providing a localised 'molecular shield' inhibiting activated $\mathrm{T}$ cells from targeting surrounding host tissues. The phenotype of PD-1 or PD-L1 knockout mice is less severe than that of CTLA-4-deficient mice (Nishimura et al, 1999; Dong et al, 2004). PD-1 knockout mice develop tissue- and strain-specific autoimmunity at approximately 9 months of age, while PD-L1 knockout mice display virtually no phenotype unless challenged with an infection or crossed onto an autoimmune prone background.

Subversion of either of these two inhibitory checkpoints is therefore a powerful mechanism by which tumours could evade host immune responses. Support for such concepts come from the demonstration of PD-L1 expression by a variety of human malignancies and correlation of expression levels with poorer overall survival. Furthermore, transfection of murine tumours with PD-L1 renders them less susceptible to lysis by cytotoxic T cells in vitro and markedly enhances tumour growth and invasiveness in vivo. More recent analyses demonstrate that in cases of PD-L1 ${ }^{+}$ melanoma, PD-L1 expression is upregulated locally within the tumour microenvironment in areas of greatest T-cell infiltration (Taube et al, 2012). More specifically, $\mathrm{PD}-\mathrm{L} 1^{+}$melanoma cells were almost always localised immediately adjacent to tumourinfiltrating lymphocytes (TILs), while tumours with minimal TIL infiltration were less likely to express $\mathrm{PD}-\mathrm{L} 1$. Interferon- $\gamma$ was detected at the interface of PD-L1 ${ }^{+}$tumours and TILs, whereas none was found in $\mathrm{PD}-\mathrm{L1}^{-}$tumours, suggesting that TILs may actually trigger their own inhibition by secreting cytokines that drive tumour PD-L1 expression, a form of adaptive resistance mediating immune escape. In other tumours, PD-L1 expression appears to be driven by constitutively active oncogenic signaling pathways (Parsa et al, 2007).

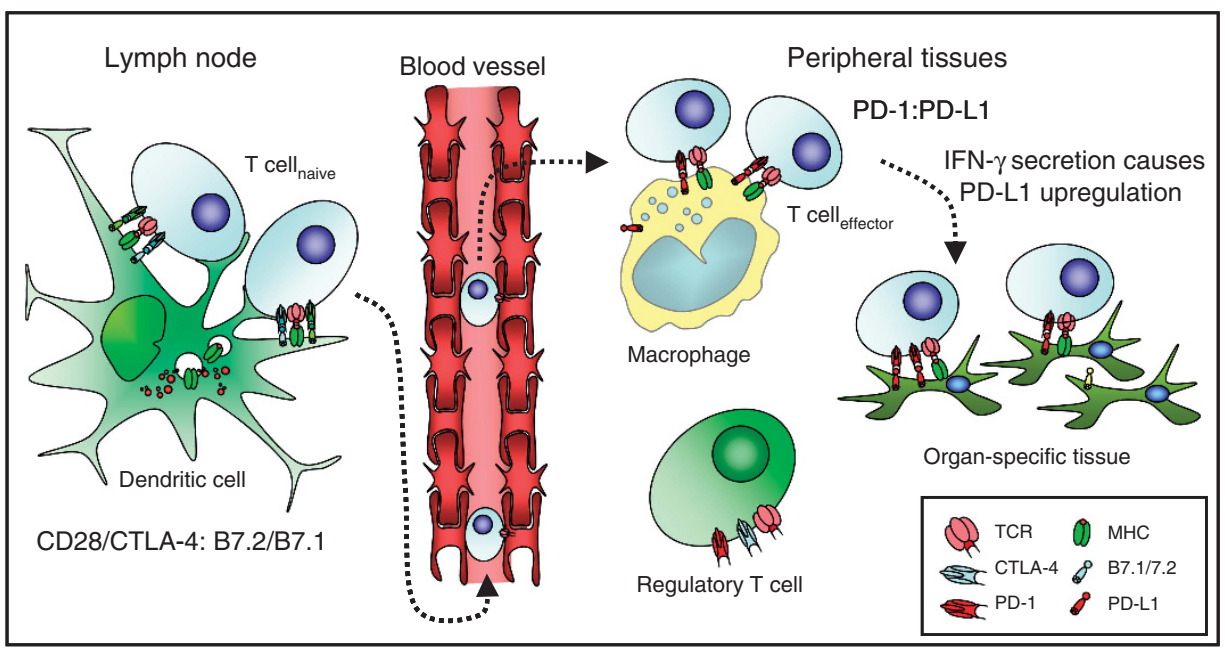

Figure 1. Compartmentalisation of co-inhibitory checkpoints. 
More direct evidence for the potential therapeutic impact of targeting these pathways came from studies in preclinical murine tumour models demonstrating enhanced anti-tumour immunity after antibody-mediated blockade of CTLA-4, PD-1 or PD-L1. These studies informed the development of similar antibodies for use in humans, and these developments form the focus of majority of this review. The precise mechanisms underpinning the enhanced anti-tumour immunity demonstrated with these antibodies remain somewhat controversial. In addition to their direct cell-intrinsic function in dampening effector T-cell, responses, both CTLA-4 and PD-1 are highly expressed on regulatory $\mathrm{T}$ cells. These molecules may have direct immune inhibitory roles when expressed in trans, and engagement of the receptors on regulatory $\mathrm{T}$ cells appears critical for maximal anti-tumour activity by antiCTLA-4 antibodies (Peggs et al, 2009b). Further dissection of the precise mechanism(s) of activity should inform the development of more effective therapeutics.

\section{CTLA-4 AND THE EVOLUTION OF THE CHECKPOINT} BLOCKADE PARADIGM

CTLA-4 is the archetypal immune regulatory checkpoint. Although the predominant mechanism by which it exerts this activity remains unclear, and in all likelihood involves multiple overlapping mechanisms, functionally it appears primarily to counteract the costimulatory activity of CD28, with which it shares its two ligands (CD80 and CD86). Its major physiological role is in the initial activation stages of both naive and memory $\mathrm{T}$ cells. CTLA-4 has a much higher overall affinity for both ligands and can therefore out-compete CD28 for binding when ligand densities are limiting. This ability is facilitated by its capacity to form extended lattice-like networks with its B7 ligands, in contrast to the likely monovalent interaction between CD28 and the ligands. Thus CTLA-4 physically excludes CD28 from the immunological synapse. Furthermore, it has also been proposed to actively deliver inhibitory signals to the $\mathrm{T}$ cell, may influence T-cell motility (Schneider et al, 2006) and may capture and strip its ligands from antigen-presenting cells by trans-endocytosis (Qureshi et al, 2011). In addition, its engagement with B7 ligands appears to deliver inhibitory signals into the ligand-expressing cells, and it also has suppressive activity in trans when expressed on either regulatory or effector T cells (Wing et al, 2008; Corse and Allison, 2012).

Early evidence for its potential as a target for enhancing antitumour immunity came from murine models (Leach et al, 1996), followed shortly thereafter by clinical evaluation of fully human anti-CTLA-4 antibodies. Objective responses as defined by Response Evaluation Criteria in Solid Tumours (RECIST) were documented in $10-15 \%$ of patients, though immune-related adverse events (IRAEs) involving a variety of tissues, including the gastrointestinal tract, were documented in $25-30 \%$ of cases treated at the higher doses (reviewed in Peggs et al, 2009a), highlighting the relatively narrow therapeutic index. Clinical responses appeared to correlate with the development of IRAEs, though this correlation was not absolute in either direction (Downey et al, 2007). Further development focused on defining the most appropriate dose and scheduling, as well as on methods to more aggressively manage IRAEs, which included budesonide and anti-TNF $\alpha$ agents, culminating in two successful phase III studies with the fully human anti-CTLA-4 monoclonal antibody ipilimumab.

The first of these trials documented outcomes in 676 patients with previously treated advanced melanoma (Hodi et al, 2010). Patients were randomised to be treated either with a peptide vaccine against the melanosomal gp100 antigen, ipilimumab or a combination of the peptide vaccine and ipilimumab. The most noteworthy outcome was a prolongation in overall survival in those treated with ipilimumab, either alone or in combination with peptide vaccination, compared with those receiving peptide vaccine monotherapy. The median survival for the ipilimumab monotherapy group was 10.1 months compared with 6.4 months for the gp100 vaccine monotherapy group $(P<0.001)$. The combination arm showed a similar outcome to ipilimumab alone (10.0 months). Furthermore, $23.5 \%$ of patients in the ipilimumab group were alive at 2 years compared with $21.6 \%$ in the combination therapy group and only $13.7 \%$ in the gp100 group. Progression-free survival did not differ significantly between the groups. The original primary end point of best overall response rate was $10.9 \%$ in the ipilimumab monotherapy group, although the 'disease control rate' (including those with stable disease over a 12 -week period) was $28.5 \%$. Of those obtaining an objective response, the response was maintained for at least 2 years in 13 out of 38 (34\%). Approximately $15 \%$ of patients treated with ipilimumab experienced grade 3-4 adverse events, and most were immune related, in keeping with the earlier phase II studies. In seven patients, these were causally associated with mortality. A significant number of those surviving 2 years had ongoing IRAEs (12 vitiligo, 4 diarrhoea or colitis and 8 endocrine requiring hormone replacement, e.g., hypopituitarism).

The results represented the first positive randomised clinical trial ever reported in patients with metastatic melanoma in terms of overall survival and the first showing a beneficial effect of a melanoma treatment in the second-line setting. Both of the other US FDA-approved drugs used in melanoma (dacarbazine and IL-2) at that time were approved without phase III clinical trial data. This trial also drew attention to the fact that immune-based therapies work in an entirely different way to most anti-cancer drugs that we have become accustomed to. The mechanisms underlying the anti-tumour activity of immunostimulatory therapies are indirect, relying on the activation of tumour-reactive immune effector cells, and contrasting with the direct activity of most conventional chemotherapeutics. The kinetics of clinical responses may therefore differ significantly, potentially taking longer to become manifest. Enhancing immunity may either deliver delayed responses, or potentially hold the tumour in a state of 'clinical equilibrium', delivering prolonged survival in the absence of objective response. Judging these therapies primarily by RECIST may therefore be inappropriate. Efforts to develop alternative immune-related response criteria (irRC) remain somewhat controversial, but further prospective validation alongside RECIST criteria, including evaluation in non immune-based therapy trials should help to establish their worth (Wolchok et al, 2009; Ribas et al, 2009; Hoos et al, 2010). One novel aspect of the irRC is the measurement of overall tumour burden as a metric of disease progression, compared with the baseline lesion measurements taken with WHO and RECIST. Although this may make good theoretical and clinical sense, it will make the process of routine reporting of imaging studies significantly more arduous. According to irRC, new lesions do not constitute disease progression if net tumour burden (including new lesions) is stable or decreases. The irRC also permit disease progression before response and introduce the concept of confirmation of progression at a subsequent timepoint after first detection. This accounts for the period required for activated $\mathrm{T}$ cells to infiltrate the tumour, which may cause initial increase in tumour volume but can subsequently translate into tumour shrinkage. The irRC also classify durable stable disease as clinical activity.

Despite the fact that the results indicated that therapeutic benefit may be confined to a minority, and that its duration may be modest in many of these, a small number did sustain remarkably prolonged responses. The difference in overall survival at 2 years in the monotherapy groups (23.5 vs 13.7\%) appeared encouraging and, while patient numbers with longer follow-up were small, 
overall survival at 4 years appeared similar in the ipilimumab group (approximately 20\%). Much effort is now being directed at identifying biomarkers that correlate with response/outcome to allow appropriate targeting of treatment to those likely to gain most benefit, both to avoid exposure to potentially harmful side effects in those unlikely to gain benefit and to manage the financial cost associated with therapy. Such insights might also allow development of strategies to enhance activity in those who are unlikely to profit from ipilimumab monotherapy.

One possible avenue for investigation in this regard is the tumour microenvironment before therapy. A subset of patients with melanoma display an inflamed tumour microenvironment associated with more effective recruitment of $\mathrm{CD}^{+}$effector $\mathrm{T}$ cells. It is tempting to speculate that the patients gaining clinical benefit from ipilimumab are among those who have an ongoing immune interaction between the tumour and the host immune system, in a similar way as can be predicted for those responding to anti-PD-1 or anti-PD-L1. Such considerations are also of importance to contextualise these data with respect to the results of adoptive T-cell therapies in melanoma. These have been dramatic in many cases with response rates of up to $50 \%$ (Dudley et al, 2008). However, successful isolation and expansion of tumour-reactive $\mathrm{T}$ cells in the early studies was possible in only approximately $30 \%$ of cases, and these are probably over-represented by those with this more 'inflamed' phenotype. Response in $50 \%$ of these cases thus translates to a response in only $15 \%$ of the overall patient population with melanoma. These considerations raise a number of important questions. Are these the same patients who would be responsive to ipilimumab? If so, will a generic off-the-shelf product be more widely applied than a patient-specific T-cell product? Although FDA approval of the first patient-specific cellular therapeutic, Sipuleucel-T, for advanced hormone-refractory prostate cancer has shown that delivery models for such therapeutics can be established, the expense and practical challenges remain formidable. Furthermore, will this mean that combination of these approaches (i.e., cellular therapy and costimulatory blockade) is unlikely to significantly increase response rates? Further exploration is clearly warranted.

Many other possible combinatorial strategies are already being investigated in preclinical models or in the clinic. As CTLA-4 and PD-1 signal through completely different mechanisms (Parry et al, 2005) and have distinct roles in regulating $\mathrm{T}$-cell responses, blockade could give additive or synergistic activity. Conversely, if tumour CD8 infiltration is a strong biomarker for response with both agents, benefits might be more modest but toxicities compounded. A current prospective trial should answer such questions. Other combinations with strong theoretical and preclinical support include combinations with anti-cancer cellular or DNA vaccines, and combinations with small-molecule inhibitors (notable given the successful development of mutant BRAF and MEK inhibitors). Combination with other conventional therapies is also being investigated. The second important randomised phase III study (placebo-controlled) of ipilimumab was in combination with dacarbazine in patients with previously untreated metastatic melanoma (Robert et al, 2011). In this trial, 502 patients were randomly assigned to receive dacarbazine with or without ipilimumab at a somewhat higher dose. Therapy was also more prolonged in this study compared with the earlier phase III study. Overall survival was significantly longer in the group receiving ipilimumab plus dacarbazine than in the group receiving dacarbazine plus placebo (11.2 months vs 9.1 months), with higher survival rates in the ipilimumab-dacarbazine group at 1 year (47.3\% vs $36.3 \%), 2$ years $(28.5 \%$ vs $17.9 \%)$ and 3 years $(20.8 \%$ vs $12.2 \%$ ). Grade $3-4$ adverse events occurred in $56.3 \%$ of patients treated with ipilimumab plus dacarbazine, as compared with $27.5 \%$ treated with dacarbazine and placebo $(P<0.001)$. No drug-related deaths occurred in the ipilimumab-dacarbazine group. The results therefore mirror those of the study in more advanced disease, establishing a role for ipilimumab in treatment-naive patients. Once again, the benefits were relatively modest in terms of prolongation of median overall survival, but a minority of patients achieved what appeared to be more durable responses. Arguably, confirmation of durability of responses in this group will ultimately offer the best insights into the true impact of ipilimumab in patients with melanoma.

\section{PD-1/PD-L1: DENTING THE MOLECULAR SHIELD}

In contrast to CTLA-4, the major role of PD-1 is thought to be the limitation of activity of $\mathrm{T}$ cells in peripheral tissues at the time of an inflammatory response to infection and to limit autoimmunity. Furthermore, chronic antigen exposure can lead to persistently high levels of PD-1 expression, which appears to induce a state of 'exhaustion' or anergy of antigen-specific T-cells, but which can be, at least partially, reversed by PD-1 blockade (Barber et al, 2006). The finding that PD-1 is expressed by a large proportion of TILs (Ahmadzadeh et al, 2009) and that PD-L1 is upregulated in a number of cancers where its expression levels often correlate with adverse outcomes, make it an attractive target for therapeutic manipulation (Dong et al, 2002). Equally, the less severe phenotype of knockout mice coupled with its lesser role in the early stages of T-cell activation raises the possibility that IRAEs might be less frequent or less severe and more likely confined to sites with 'inflammatory' signatures.

The early clinical experience of large phase I studies exploring the use of anti-PD-1 or anti-PD-L1 monoclonal antibodies has received considerable attention. The anti-PD-1 study detailed outcomes in 296 patients with previously treated advanced cancers, including melanoma, renal cell cancer, non-small-cell lung cancer (NSCLC), castration-resistant prostate cancer and colorectal cancer (Topalian et al, 2012). Patients received anti-PD-1 antibody intravenously every 2 weeks in escalating dose cohorts, with treatment duration up to 12 cycles. Encouraging responses were documented in melanoma (28\%), renal cell carcinoma (27\%) and, perhaps more unexpectedly, NSCLC (18\%). No objective responses were observed in the relatively small cohorts with prostate or colon cancer. As with ipilimumab, responses appeared durable in a number of cases (lasting over 12 months in 20 patients at the latest follow-up). A subset of tumours were analysed for PD-L1 expression. In keeping with the proposed mechanism of action, none of those with PD-L1-negative tumours $(n=17$, including 9 with colorectal or prostate cancer) achieved an objective response, compared with 9 out of 25 (36\%) with PD-L1-positive tumours. Adverse events consistent with immune-mediated causes were observed in $41 \%$ of patients. Grade 3-4 immune-related events occurred in only $6 \%$, although fatal pneumonitis was seen in three cases.

As this was a phase 1 study with multiple escalation cohorts, it is difficult to be certain of the overall response rates, which could be underestimated at lower doses. Nevertheless, the response rates in melanoma looked impressive in relation to the early- and latephase results with CTLA-4 blockade. The responses in NSCLC, though less frequent, are in some ways more surprising and impressive. Objective responses were noted in 14 out of 76 (18\%) 'evaluable' patients, and 13 out of 58 (22\%) treated at dose levels of $3.0-10.0 \mathrm{mg} \mathrm{kg}^{-1}$ (although it is unclear why 46 out of 122 of such patients were considered non-evaluable). Eight out of 14 such responses were documented to last $\geqslant 24$ weeks. These results require confirmation but point to a valuable therapeutic advance in this difficult patient group. 
In terms of the proposed mechanism of action, the data on responsiveness and PD-L1 expression are clearly provocative. As $98 \%$ of PD-L1-positive tumours were associated with TILs compared with only $28 \%$ of $\mathrm{PD}$-L1-negative tumours, the majority of human melanoma lesions fall into one of the three distinct categories: those that are $\mathrm{PD}-\mathrm{L}^{+}{ }^{+}$and $\mathrm{TIL}^{+}$, which will likely contain the vast majority of cases responsive to PD-1 (or PD-L1) blockade; those that are $\mathrm{PD}-\mathrm{L}^{-}{ }^{-}$and $\mathrm{TIL}^{+}$, which are unlikely to respond to PD-L1 (and probably PD-1) blockade, but in which other co-inhibitory pathways may be more relevant and potential targets for manipulation (e.g., CTLA-4); and those that are PD$\mathrm{L} 1^{-}$and $\mathrm{TIL}^{-}$, which are perhaps unlikely to respond to manipulation of co-inhibitory circuits. Although such analysis may provide good biomarkers to allow targeting of therapies to appropriate patient groups and to improve apparent response rates, it is important to remain aware that patients with $\mathrm{TIL}^{+}$tumours and favourable effector/regulatory ratios tend to have better prognoses in any case, presumably reflective of the impact of ongoing immune surveillance on tumour outgrowth. It seems likely that the $\mathrm{PD}-\mathrm{L}^{-}$and $\mathrm{TIL}^{-}$subgroup will present the greatest therapeutic challenge in terms of manipulation of immune inhibitory checkpoints. A further layer of complexity is added by consideration of possible intratumoural heterogeneity in PD-L1 expression and TIL infiltration, which may require evaluation with multiple biopsies from the same individual.

With regards to toxicity, it is notable that the overall incidence of adverse events with probable immune aetiology was not much different in the anti-PD-1 study than the ipilimumab studies but that the severity did appear more modest in the majority. Interpretation is, however, confounded by the propagation of an increased awareness of the potential for such toxicity issues and more aggressive intervention with immune suppressants, for example, glucocorticoids. The cases of fatal pneumonitis are clearly of some concern, potentially restricting application of anti-PD-1 in both the adjuvant and the neo-adjuvant settings. In this regard, it is interesting to note that the lung is infiltrated by relatively high levels of PD-L1-expressing antigen-presenting cells and that this may have relevance with respect to this organ-specific toxicity. Nevertheless, this may also be of relevance to the responses demonstrated for both squamous and non-squamous NSCLCs. It is clear that future combinatorial approaches evaluating agents targeting multiple checkpoints, for example, anti-PD-1 and antiCTLA-4, will need very careful titration of both agents to avoid additive or multiplicative toxicities.

A parallel phase I study investigated the use of anti-PD-L1 in a similar patient cohort (Brahmer et al, 2012). Although response rates looked superficially less compelling (melanoma 17\%, renal cell carcinoma $12 \%$, NSCLC 10\%), the current data cannot answer the question as to which approach is preferable. Pre-clinical studies have generally indicated a greater impact of PD-L1 blockade, and specific antibody properties or dosing issues may be relevant to the phase I clinical studies given the widespread distribution of PD-L1. Blockade of receptor or ligand would not necessarily be expected to be equivalent because of the complexity of the $\mathrm{PD}-1$ receptor/ ligand axis. PD-L1 binds to CD80 (B7.1) with an affinity greater than CD28 (Butte et al, 2007). Thus an anti-PD1 antibody would block PD-1 from interacting with PD-L1 and PD-L2 but not the interaction with PD-L1 and CD80 (potentially sequestering CD80 from exerting a co-stimulatory signal through CD28, as well as mediating direct inhibitory signals). By contrast, an anti-PD-L1 antibody would block the interaction between PD-L1 and CD80 as well as between PD-L1 and PD-1 but would not block the interaction between PD-1 and PD-L2. The ability of the ligands to back-signal into the cell expressing them (in many cases delivering inhibitory signals) adds a further level of complexity when considering blocking antibodies. Finally, the ability of such antibodies to mediate agonistic signalling is also relatively poorly understood.

\section{CONCLUSIONS}

Recent years have provided ample evidence that manipulation of the co-inhibitory pathways that restrict anti-tumour immunity can remove the immunological brakes that impede the induction of clinically meaningful anti-tumour responses even when administered as monotherapies. The next few years should see the larger confirmatory phase 3 studies of anti-PD-1 in a number of malignancies (likely melanoma, renal cell carcinoma and NSCLC). These will give a better indication of the toxicity profile, which, on first impression, appears favourable compared with that of antiCTLA-4 antibodies. Correlation with TIL and PD-L1 status will also provide invaluable insights into appropriate targeting of such therapies. We will also see the results of early combinatorial studies, including those of conventional therapies with anti-PD-1, and of anti-PD-1 with other immunotherapies, notably antiCTLA-4. Furthermore, it is noteworthy that the extended immunoglobulin superfamily includes other apparently inhibitory ligands for which the receptors are yet to be defined, that is, B7-H3 and B7-H4 (Zang et al, 2003; Yi and Chen, 2009; Zang et al, 2010). These offer further possible therapeutic targets, particularly as their expression profiles are non-overlapping with PD-L1 in some malignancies. Finally, other inhibitory receptors, including Tim3 and LAG-3, can be upregulated on TILs establishing further combinatorial opportunities (Sakuishi et al, 2010; Goldberg and Drake, 2011; Woo et al, 2012). Although the overall winners in terms of the best target(s) among these multiple possibilities will require careful clinical evaluation, supported by dissection of mechanism, it is perhaps more pertinent to reflect that the true winners are likely to be the patients. Discussion will no doubt focus more on cost implications over coming years, but this is welcome progress. We can now move on from discussions more heavily centred on whether immunotherapies based on enhancing preexisting patient anti-tumour immunity will ever have a role in the treatment of cancer to how these will be best integrated into patient treatment pathways.

\section{REFERENCES}

Ahmadzadeh M, Johnson LA, Heemskerk B, Wunderlich JR, Dudley ME, White DE, Rosenberg SA (2009) Tumor antigen-specific CD8 T cells infiltrating the tumor express high levels of PD-1 and are functionally impaired. Blood 114: 1537-1544.

Barber DL, Wherry EJ, Masopust D, Zhu B, Allison JP, Sharpe AH, Freeman GJ, Ahmed R (2006) Restoring function in exhausted CD8 T cells during chronic viral infection. Nature 439: 682-687.

Brahmer JR, Tykodi SS, Chow LQ, Hwu WJ, Topalian SL, Hwu P, Drake CG, Camacho LH, Kauh J, Odunsi K, Pitot HC, Hamid O, Bhatia S, Martins R, Eaton K, Chen S, Salay TM, Alaparthy S, Grosso JF, Korman AJ, Parker SM, Agrawal S, Goldberg SM, Pardoll DM, Gupta A, Wigginton JM (2012) Safety and activity of anti-PD-L1 antibody in patients with advanced cancer. $N$ Engl J Med 366: 2455-2465.

Burnet FM (1967) Immunological aspects of malignant disease. Lancet 1: 1171-1174.

Butte MJ, Keir ME, Phamduy TB, Sharpe AH, Freeman GJ (2007) Programmed death-1 ligand 1 interacts specifically with the B7-1 costimulatory molecule to inhibit $\mathrm{T}$ cell responses. Immunity 27: 111-122.

Chambers CA, Sullivan TJ, Allison JP (1997) Lymphoproliferation in CTLA4-deficient mice is mediated by costimulation-dependent activation of CD4 + T cells. Immunity 7: 885-895.

Corse E, Allison JP (2012) Cutting edge: CTLA-4 on effector T cells inhibits in trans. J Immunol 189: 1123-1127.

Dong H, Strome SE, Salomao DR, Tamura H, Hirano F, Flies DB, Roche PC, Lu J, Zhu G, Tamada K, Lennon VA, Celis E, Chen L (2002) 
Tumor-associated B7-H1 promotes T-cell apoptosis: a potential mechanism of immune evasion. Nat Med 8: 793-800.

Dong H, Zhu G, Tamada K, Flies DB, van Deursen JM, Chen L (2004) B7-H1 determines accumulation and deletion of intrahepatic $\mathrm{CD} 8(+) \mathrm{T}$ lymphocytes. Immunity 20: 327-336.

Downey SG, Klapper JA, Smith FO, Yang JC, Sherry RM, Royal RE, Kammula US, Hughes MS, Allen TE, Levy CL, Yellin M, Nichol G, White DE, Steinberg SM, Rosenberg SA (2007) Prognostic factors related to clinical response in patients with metastatic melanoma treated by CTL-associated antigen-4 blockade. Clin Cancer Res 13: 6681-6688.

Dudley ME, Yang JC, Sherry R, Hughes MS, Royal R, Kammula U, Robbins PF, Huang J, Citrin DE, Leitman SF, Wunderlich J, Restifo NP, Thomasian A, Downey SG, Smith FO, Klapper J, Morton K, Laurencot C, White DE, Rosenberg SA (2008) Adoptive cell therapy for patients with metastatic melanoma: evaluation of intensive myeloablative chemoradiation preparative regimens. J Clin Oncol 26: 5233-5239.

Goldberg MV, Drake CG (2011) LAG-3 in cancer immunotherapy. Curr Top Microbiol Immunol 344: 269-278.

Hodi FS, O’Day SJ, McDermott DF, Weber RW, Sosman JA, Haanen JB, Gonzalez R, Robert C, Schadendorf D, Hassel JC, Akerley W, van den Eertwegh AJ, Lutzky J, Lorigan P, Vaubel JM, Linette GP, Hogg D, Ottensmeier CH, Lebbe C, Peschel C, Quirt I, Clark JI, Wolchok JD, Weber JS, Tian J, Yellin MJ, Nichol GM, Hoos A, Urba WJ (2010) Improved survival with ipilimumab in patients with metastatic melanoma. N Engl J Med 363: 711-723.

Hoos A, Eggermont AM, Janetzki S, Hodi FS, Ibrahim R, Anderson A, Humphrey R, Blumenstein B, Old L, Wolchok J (2010) Improved endpoints for cancer immunotherapy trials. J Natl Cancer Inst 102: 1388-1397.

Koebel CM, Vermi W, Swann JB, Zerafa N, Rodig SJ, Old LJ, Smyth MJ, Schreiber RD (2007) Adaptive immunity maintains occult cancer in an equilibrium state. Nature 450: 903-907.

Leach DR, Krummel MF, Allison JP (1996) Enhancement of antitumor immunity by CTLA-4 blockade. Science 271: 1734-1736.

Nishimura H, Nose M, Hiai H, Minato N, Honjo T (1999) Development of lupus-like autoimmune diseases by disruption of the PD-1 gene encoding an ITIM motif-carrying immunoreceptor. Immunity 11: 141-151.

Parry RV, Chemnitz JM, Frauwirth KA, Lanfranco AR, Braunstein I, Kobayashi SV, Linsley PS, Thompson CB, Riley JL (2005) CTLA-4 and PD-1 receptors inhibit T-cell activation by distinct mechanisms. Mol Cell Biol 25: 9543-9553.

Parsa AT, Waldron JS, Panner A, Crane CA, Parney IF, Barry JJ, Cachola KE, Murray JC, Tihan T, Jensen MC, Mischel PS, Stokoe D, Pieper RO (2007) Loss of tumor suppressor PTEN function increases B7-H1 expression and immunoresistance in glioma. Nat Med 13: 84-88.

Peggs KS, Quezada SA, Allison JP (2009a) Cancer immunotherapy: co-stimulatory agonists and co-inhibitory antagonists. Clin Exp Immunol 157: 9-19.

Peggs KS, Quezada SA, Chambers CA, Korman AJ, Allison JP (2009b) Blockade of CTLA-4 on both effector and regulatory T cell compartments contributes to the antitumor activity of anti-CTLA-4 antibodies. J Exp Med 206: 1717-1725.

Qureshi OS, Zheng Y, Nakamura K, Attridge K, Manzotti C, Schmidt EM, Baker J, Jeffery LE, Kaur S, Briggs Z, Hou TZ, Futter CE, Anderson G, Walker LS, Sansom DM (2011) Trans-endocytosis of CD80 and CD86: a molecular basis for the cell-extrinsic function of CTLA-4. Science 332: 600-603.
Ribas A, Chmielowski B, Glaspy JA (2009) Do we need a different set of response assessment criteria for tumor immunotherapy? Clin Cancer Res 15: 7116-7118.

Robert C, Thomas L, Bondarenko I, O’Day S, JW MD, Garbe C, Lebbe C, Baurain JF, Testori A, Grob JJ, Davidson N, Richards J, Maio M, Hauschild A, Miller Jr. WH, Gascon P, Lotem M, Harmankaya K, Ibrahim R, Francis S, Chen TT, Humphrey R, Hoos A, Wolchok JD (2011) Ipilimumab plus dacarbazine for previously untreated metastatic melanoma. N Engl J Med 364: 2517-2526.

Sakuishi K, Apetoh L, Sullivan JM, Blazar BR, Kuchroo VK, Anderson AC (2010) Targeting Tim-3 and PD-1 pathways to reverse T cell exhaustion and restore anti-tumor immunity. J Exp Med 207: 2187-2194.

Schneider H, Downey J, Smith A, Zinselmeyer BH, Rush C, Brewer JM, Wei B, Hogg N, Garside P, Rudd CE (2006) Reversal of the TCR stop signal by CTLA-4. Science 313: 1972-1975.

Shankaran V, Ikeda H, Bruce AT, White JM, Swanson PE, Old LJ, Schreiber RD (2001) IFNgamma and lymphocytes prevent primary tumour development and shape tumour immunogenicity. Nature 410: 1107-1111.

Taube JM, Anders RA, Young GD, Xu H, Sharma R, McMiller TL, Chen S, Klein AP, Pardoll DM, Topalian SL, Chen L (2012) Colocalization of inflammatory response with B7-h1 expression in human melanocytic lesions supports an adaptive resistance mechanism of immune escape. Sci Transl Med 4: 127ra37.

Topalian SL, Hodi FS, Brahmer JR, Gettinger SN, Smith DC, McDermott DF, Powderly JD, Carvajal RD, Sosman JA, Atkins MB, Leming PD, Spigel DR, Antonia SJ, Horn L, Drake CG, Pardoll DM, Chen L, Sharfman WH, Anders RA, Taube JM, McMiller TL, Xu H, Korman AJ, Jure-Kunkel M, Agrawal S, McDonald D, Kollia GD, Gupta A, Wigginton JM, Sznol M (2012) Safety, activity, and immune correlates of anti-PD-1 antibody in cancer. N Engl J Med 366: 2443-2454.

Wing K, Onishi Y, Prieto-Martin P, Yamaguchi T, Miyara M, Fehervari Z, Nomura T, Sakaguchi S (2008) CTLA-4 control over Foxp3 + regulatory T cell function. Science 322: 271-275.

Wolchok JD, Hoos A, O’Day S, Weber JS, Hamid O, Lebbe C, Maio M, Binder M, Bohnsack O, Nichol G, Humphrey R, Hodi FS (2009) Guidelines for the evaluation of immune therapy activity in solid tumors: immunerelated response criteria. Clin Cancer Res 15: 7412-7420.

Woo SR, Turnis ME, Goldberg MV, Bankoti J, Selby M, Nirschl CJ, Bettini ML, Gravano DM, Vogel P, Liu CL, Tangsombatvisit S, Grosso JF, Netto G, Smeltzer MP, Chaux A, Utz PJ, Workman CJ, Pardoll DM, Korman AJ, Drake CG, Vignali DA (2012) Immune inhibitory molecules LAG-3 and PD-1 synergistically regulate T-cell function to promote tumoral immune escape. Cancer Res 72: 917-927.

Yi KH, Chen L (2009) Fine tuning the immune response through B7-H3 and B7-H4. Immunol Rev 229: 145-151.

Zang X, Loke P, Kim J, Murphy K, Waitz R, Allison JP (2003) B7x: a widely expressed B7 family member that inhibits T cell activation. Proc Natl Acad Sci USA 100: 10388-10392.

Zang X, Sullivan PS, Soslow RA, Waitz R, Reuter VE, Wilton A, Thaler HT, Arul M, Slovin SF, Wei J, Spriggs DR, Dupont J, Allison JP (2010) Tumor associated endothelial expression of $\mathrm{B} 7-\mathrm{H} 3$ predicts survival in ovarian carcinomas. Mod Pathol 23: 1104-1112.

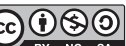

This work is licensed under the Creative Commons Attribution-NonCommercial-Share Alike 3.0 Unported License. To view a copy of this license, visit http://creativecommons. org/licenses/by-nc-sa/3.0/ 